\title{
LIGHT POLLUTION IN LEO-SATELLITE TRACKING
}

\author{
L.S.Shakun ${ }^{1}$, V.I.Marsakova ${ }^{2}$, T.A.Golubovskaya ${ }^{1}$, S.S.Terpan ${ }^{1}$, \\ S.M.Melikyants ${ }^{1}$, E.A.Korobeynikova ${ }^{1}$ \\ ${ }^{1}$ Astronomical Observatory of I.I.Mechnikov Odessa National University \\ ${ }^{2}$ Astronomy Department of I.I.Mechnikov Odessa National University \\ Shevchenko Park, Odessa 65014, Ukraine \\ leomspace@gmail.com,v.marsakova@onu.edu.ua
}

\begin{abstract}
Because of the high level of the light pollution in Odessa city, only the observations of LEO (low Earth orbit)-satellites are performed in the main office of the Astronomical observatory of Odessa National University named after I.I. Mechnikov. As the one of the observation results we obtain the sky background measurements along the satellite's way through the sky (at different azimuths and altitudes that change during the satellite tracking). We propose the method of irregular extinction changes diagnostics that realized by using the different data filtration methods. Also as the result of our analysis of these observations we present azimuth-altitude diagram of sky background that shows the most significant light pollution at the north-western and northern directions caused by the Port of Odessa and the stadium "Chernomorets".
\end{abstract}

Key words: Sky background; light pollution; LEOsatellites: optical observations: reduction; LEO-satellites: photometry.

\section{Observations}

Observatory of I.I.Mechnikov Odessa National University that located near historical center of Odessa, in the T.G.Shevchenko park. Thus, only observations of the LEO (Low Earth Orbit) satellites still take place there. The satellites are bright enough and they are observed with relatively short expositions (because of their fast motion) to make the coordinate measurements and the photometry possible.

We perform the observations by using telescope KT-50 with analog CCD-camera WATEC-902H2. The diameter of the main mirror is $50 \mathrm{~cm}$, the focal length is $2 \mathrm{~m}$, the resolution and the field of view on the camera are $0,9 \mathrm{arcsec} / \mathrm{pixel}$ and 10 arcmin, consequently. The telescope works in the tracking mode along the satellite's trajectory in the sky.

There are two main aims of these observations: determination of precise positions of the satellites for refinement of their orbits and photometry for determination of their rotational parameters (Shakun \& Koshkin, 2013; Shulga et al., 2015). An example of the light variations of the satellite during one track is shown at Fig. 1. Cyclic variations due to rotation is visible.

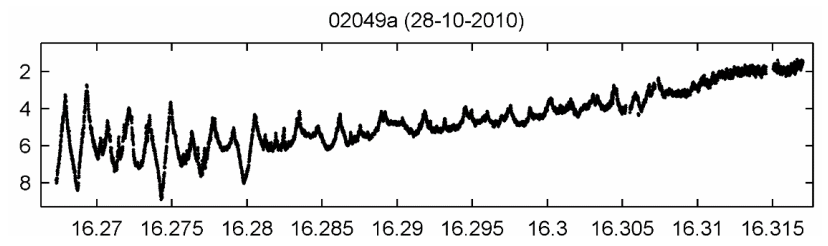

Figure 1: An example of the light variations of the satellite during one track. On horizontal axis is the time in hours, on vertical axis is the brightness in visual magnitudes.

\section{Sky background measurement}

As the one of the observation results, we obtain the sequence of images (frames) along the line of satellite way in the sky with intervals between the frames of $0.02 \mathrm{sec}$. In one clear night we obtain from 10 to 30 satellite tracks. Satellite tracking started in the twilight but we use the observations since the altitude of the Sun is not higher than 14 degrees (this limits was found experimentally, see Fig. 2, for example). The calculation of brightness (here and below) was performed in grayscale units from 0 to 250 .

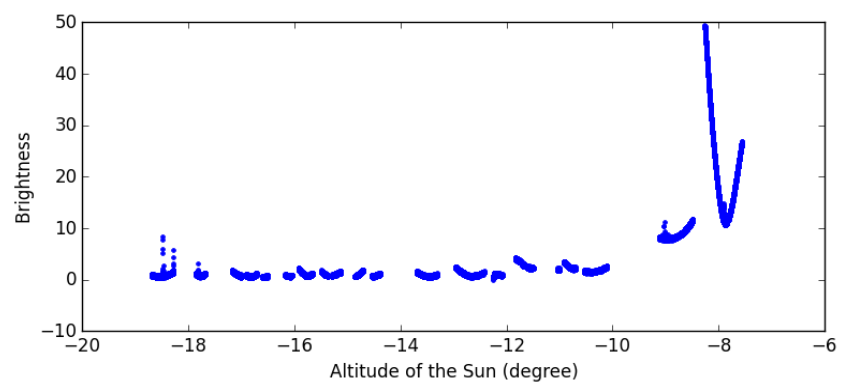

Figure 2: The observed brightness of the sky background in dependence on the altitude of the Sun (12-06-2015). 
In this work we studied 647 tracks of satellites during 47 clear nights within 7 months of this year, but the described analysis may be applied to observations in any time interval.

For each image we eliminated all pixels that have brightness higher than certain level (they were marked as objects or stars) and in the rest part of image we calculated average value of the sky background by using the each fourth pixel. Thus, we obtained the average value of the background (average of 100000 pixels) in each frame. Also for each frame the standard deviation of the background was calculated.

\section{Detection of sky background irregularities}

One of the our purposes is the sorting of tracks into "good" and "bad", because the sky can have the irregularities of the background due to an atmospheric haze, cirrus clouds and other effects that are not visible for observers. To solve this problem we applied the comparison of the smoothing curves obtained by using the methods of "running average" and "median filter" or "running median". First the strongly deviating values were discarded by using "running median". Then the curves of "running average" (with the bigger filter width of 2000 frames) and of "running median" (with the filter width of 50 frames) were compared. The deviations between these curves are shown at Fig. 5. In most cases, the value of deviations lies within from -0.07 to 0.07 . (The calculation of brightness was performed in grayscale units from 0 to 250). Therefore, the limit of 0.08 was chosen. If the maximal difference in the track had been higher of this limit then the background changes were considered as "irregular" and the track was refused as "bad". Figures 3-5 illustrate the process of discarding the strongly deviating values of the background and rejection of the "bad" tracks. Fig. 6 gives us the example of the "bad" track.

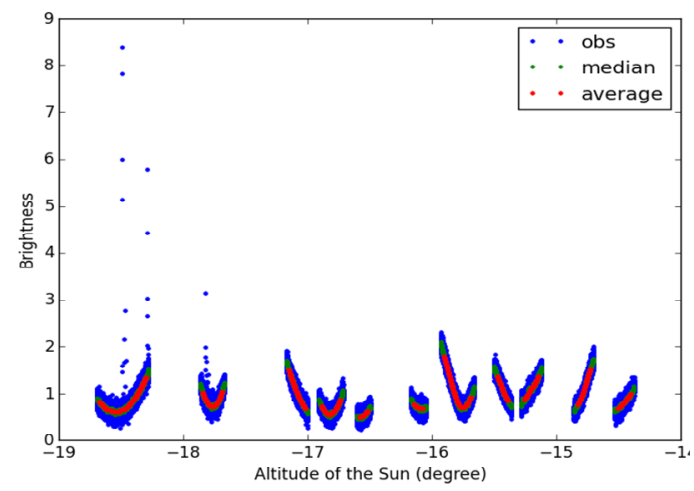

Figure 3: Initial observations of the sky background brightness without previous filtration of random deviations. All tracks on 12-06-2015.

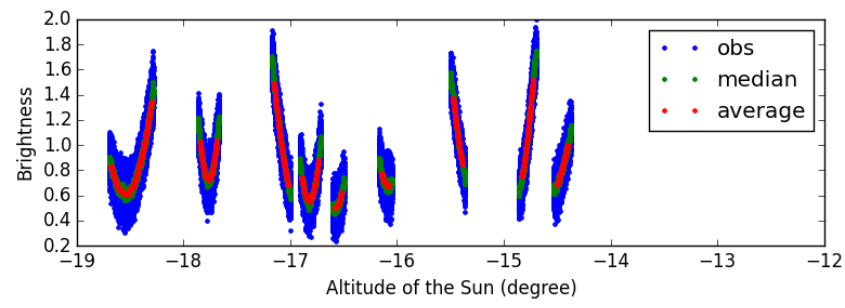

Figure 4: Brightness of the sky background after filtration of random deviations and rejection of the "bad" tracks with irregular changes of the sky background. "Good" tracks on 12-06-2015.

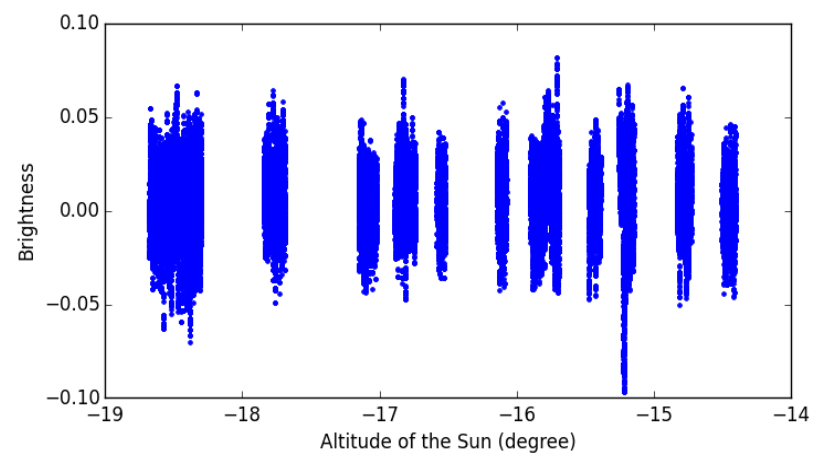

Figure 5: Difference between the approximations of the sky background by using "running average" (with the bigger filter width of 2000 frames) and of "running median" (with the filter width of 50 frames). All tracks on 12-06-2015.

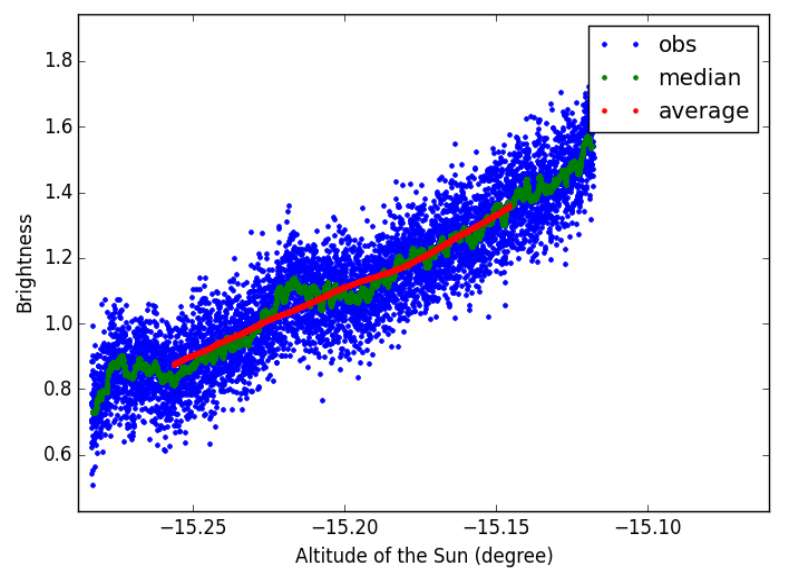

Figure 6: One of the "bad" track with irregular changes of the sky background on 12-06-2015.

\section{Distribution of the background brightness}

Our second task was the obtaining of distribution of the background brightness on the sky and determination of its azimuthal dependence for optimization of the observing program. This is important because intense light pollution, small field of view and stretching of stars during the satellite tracking can severely restrict the limiting magnitude of the telescope (In our case the limiting magnitude is about $10.5^{\mathrm{m}}$ ).

As one can see at Fig. 7, the satellite tracks cover a small percentage of the sky hemisphere area. So we gathered the values of sky background brightness in the areas of 25 square degrees (Fig. 8) using the observations within several clear nights with the abundance of the observed tracks. To avoid the discrepancy in background brightness on different dates, we built the dependence of the background brightness of one night on the analogous value of another night. This procedure was performed for all areas, which correspond to the observations on the both dates. The dependence can be well described as linear one (see examples at Fig. 9, 9a) and we calculated the transition coefficients for all nights to transform their background brightness to the level of the one chosen night (12-062015 in our example). 


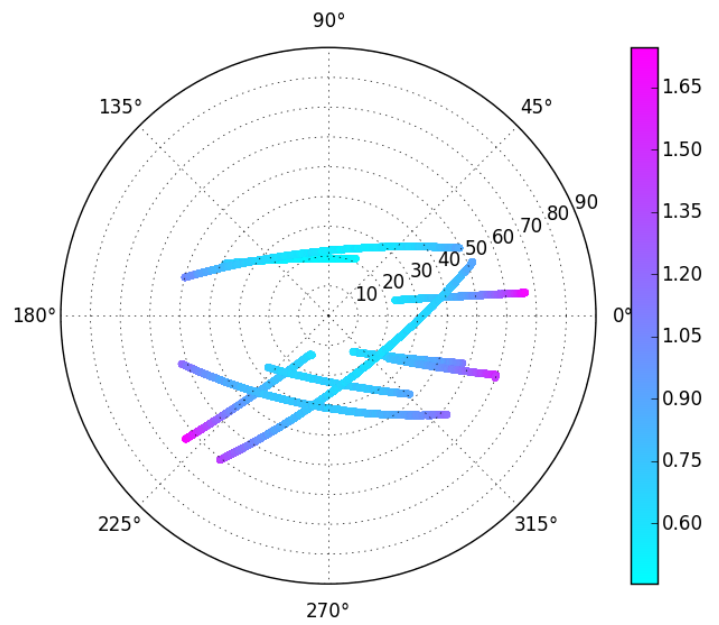

Figure 7: Sky background brightness in the azimuthal coordinates along the satellites tracks. The values of the sky background brightness are shown by using different colors. "Good" tracks on the date 12-06-2015). Zenith distances is marked on the circles.

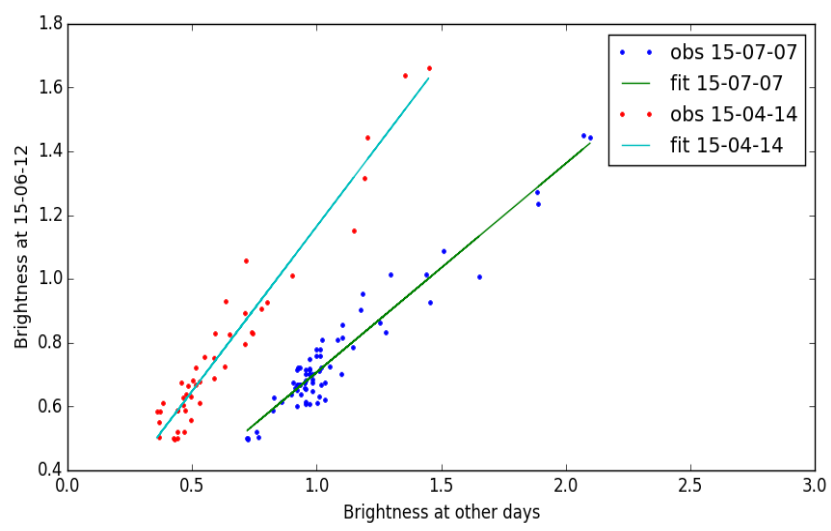

Figure 9: Sky background brightness in the same areas obtained in different "good" nights. comparison of the 14-04-2015, 07-07-2015 with12-06-2015.
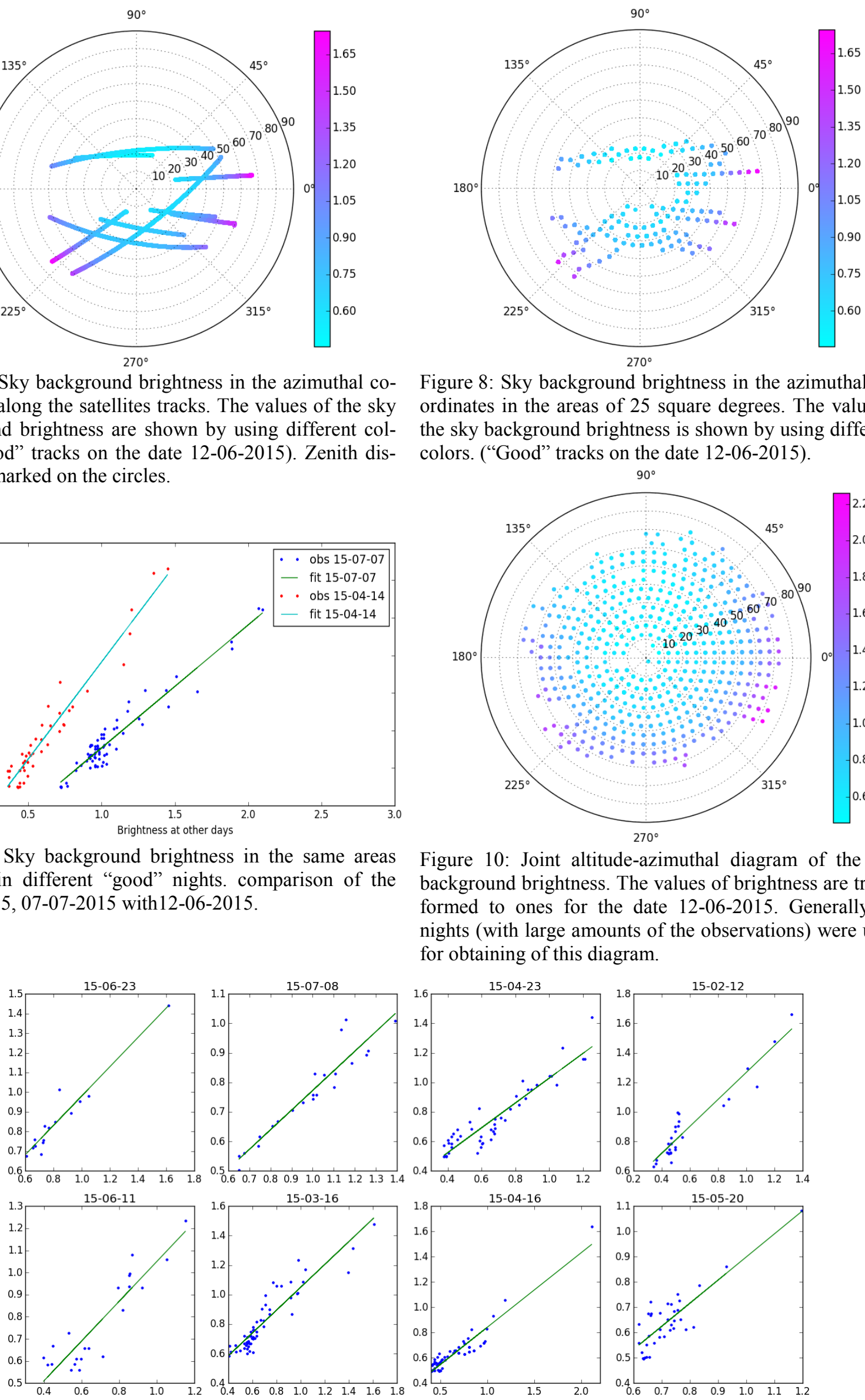

Figure 8: Sky background brightness in the azimuthal coordinates in the areas of 25 square degrees. The value of the sky background brightness is shown by using different colors. ("Good" tracks on the date 12-06-2015).

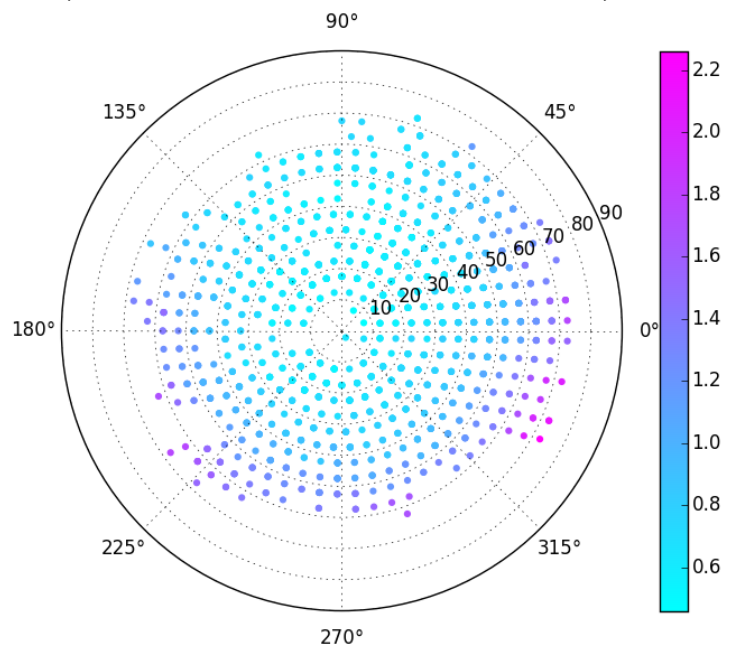

Figure 10: Joint altitude-azimuthal diagram of the sky background brightness. The values of brightness are transformed to ones for the date 12-06-2015. Generally 11 nights (with large amounts of the observations) were used for obtaining of this diagram.
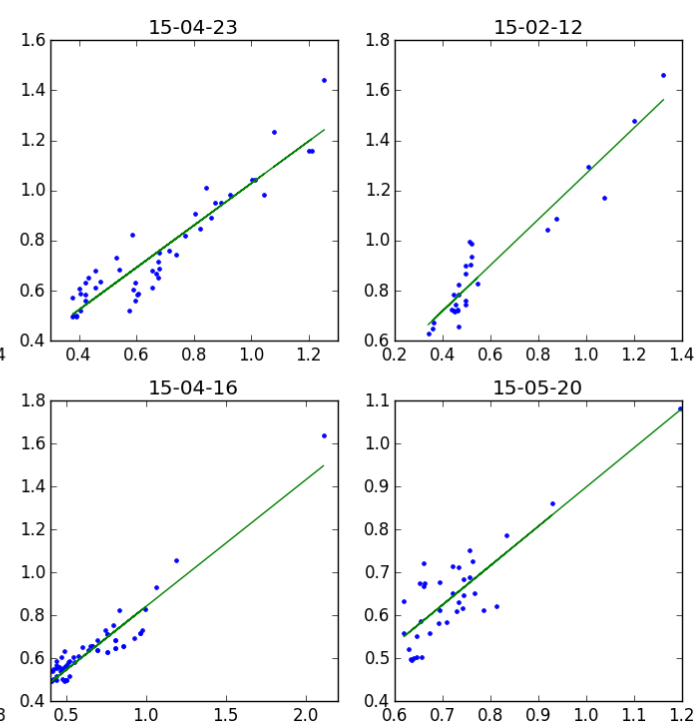

Figure 9a: Some more nights (with sufficient quantities of the observations) in comparison with the 12-06-2015. (See description of the Fig. 9) 
Finally, we obtained the joint altitude-azimuthal diagram which show the distribution of the background brightness on the sky (Fig. 10). It shows the significant increase of the light pollution (by the factor 3) in the north-western direction and minimal values of light pollution in the eastern and south-eastern directions. Since we selected the tracks in which the altitude of the Sun was not higher than -14 degrees and satellites were not observed at too low altitudes, it can't be due to the illumination from the Sun on the West side. Most likely, it is light pollution due to the activities of the Odessa Sea Port (in the Northwest) and the Stadium "Chernomorets" (in the North). East side (in the direction to the sea away from the Port) is mostly free from light pollution.

\section{Discussion}

The method of choosing of "good" tracks (with the regular changes of sky background) is based on the comparison of "running" approximations with narrow and wide filter widths. The main advantage of the method is that the information about the sky background brightness distribution or about transparency of the atmosphere in different directions is not needed a priory. This allows to process a large range of observations that were obtained in different observational conditions. But this approach has a range of certain disadvantages.

Typically the choice of the value for the smaller filter width is not difficult and it can be selected within wide limits. The choice of the value for the larger filter width is associated with a number of difficulties. Firstly, it should be significantly larger than the smaller one and that imposes restrictions on the smaller filter width. Secondly, larger filter width should be significantly wider than the size of irregularities of the sky background of which we want to detect. As such irregularities can vary in a wide range covering the significant part of the size of the track, thus the filter width tends to size comparable to the length of the entire track.

Using the running approximation with a given filter width we either lose the edges of the observation range within of filter half-width, either we deal with edge effects of such smoothing. In our case, the presence of edge effects was very undesirable, as well as the discarding of observa- tions. Thus, the larger filter width was limited by the considerations about the saving of the possible largest number of observations. Therefore, the maximum filter width must be significantly less than the length of the track. The characteristic form of the changes in sky background brightness along the track leads us to the same conclusion.

In case of large zenith distances (more than 70 degrees) the sizes of irregularities (due to both natural reasons and anthropogenic light pollution) become smaller. So it will cause discarding the whole track which cover the low altitudes of the satellite if we use excessively large filter width but that is undesirable.

Thus, we see that there are certain difficulties with the choice of the larger filter width.

The problems described above could be avoided if instead of the low-frequency smoothing we use an approximating model for the brightness of the entire sky. The making of such a model in any case requires a preliminary study of altitude-azimuthal distribution of the sky background brightness, and this task was also performed in this paper.

\section{Conclusion}

Thus, the proposed method allows to detect irregular deviations in low-frequency changes of the sky background and it enables automatic selecting of the tracks which were carried on through the cloud cover. So it makes the separation of "good" tracks (suitable for the precise photometry) possible.

Also we propose the method for building of the average light pollution map based on the entire season of LEOsatellite observations. This promotes the preparation of the optimal observations programme.

Acknowledgements. Authors are grateful to N.I.Koshkin for helpful discussions.

\section{References}

Shakun L.S., Koshkin N.I.: 2013, Advances in Space Research, 53, 1834.

Shulga V. et al.: 2015, Space Science and Technology, 21 (3), 74. 